\title{
К ВОПРОСУ О ДОСТУПНОСТИ КРЕДИТНЫХ РЕСУРСОВ ДЛЯ МАЛОГО И СРЕДНЕГО БИЗНЕСА
}

\section{ON THE ISSUE OF AVAILABILITY OF CREDIT RESOURCES FOR SMALL AND MEDIUM-SIZED BUSINESSES}

A. Ushanov

Summary. The phenomena of stagnation in the segment of small and medium-sized businesses observed in the Russian economy in recent years determine the importance of supporting the development of the sector at the legislative level, as well as improving the mechanism of access of SMEs to borrowed credit resources of banks. State regulation and support for small and medium-sized businesses, in particular, the program of subsidizing Bank rates, has not yet earned one hundred percent, first, because of the difficulty for banks with a basic license to build sustainable models of lending to small businesses, and secondly, due to the lack of development of the SME sector as a result of a number of constraints. In order to overcome the problems, the article suggests a number of corrective measures both in terms of improving legislation in this area and in relation to the practice of lending by banks to SMEs.

Keywords: small and medium-sized businesses, government regulation and support, interest rate subsidies, SME lending mechanism.

\author{
Уианов Александр Евгеньевич \\ К.э.н., доцент, Финансовый университет при \\ Правительстве РФ (г. Москва) \\ Ushanov_0656@mail.ru
}

Аннотация. Явления стагнации в сегменте малого и среднего предпринимательства, наблюдающиеся в российской экономике последние годы, предопределяют значимость поддержки развития сектора на законодательном уровне, а также совершенствования механизма доступности предприятий МСП к заемным кредитным ресурсам банков. Государственное регулирование и поддержка малого и среднего бизнеса, в частности, программа субсидирования банковских ставок, не заработало пока на сто процентов, во-первых, из-за сложности для банков с базовой лицензией выстраивать устойчивые модели кредитования малого бизнеса, во-вторых, в силу еще недостаточного развития сектора МСП в результате ряда сдерживающих факторов. В целях преодоления проблем в статье предлагается ряд корректирующих мер как в части совершенствования законодательства в данной области, так и в отношении практики кредитования банками предприятий МСП.

Ключевые слова: малое и среднее предпринимательство, государственное регулирование и поддержка, субсидирование процентных ставок, механизм кредитования МСП.

Если в 2018 г. доля малого и среднего бизнеса в экономике составила 21,9\% [1], то по итогам 2019 г. планировалось довести ее до 22,9\% (для сравнения: в Великобритании - 51\%, в Германии - 53\%, в Финляндии - 60\%, в Нидерландах - 63\%). Занятость в секторе МСП составляет $25 \%$ от числа экономически активного населения. Эти цифры последние годы остаются практически неизменными [2].

Насколько эффективны разработанные проекты и принятые программы - покажет время, но сегодня в данной области над позитивными тенденциями явно превалируют отрицательные. Так, по итогам третьего квартала 2019 года малый и средний бизнес России приблизился к состоянию стагнации - значение индекса деловой активности малых и средних предприятий (RSBI) снизилось до 50,9 пункта [3]. Проведенная Счетной палатой «Оценка состояния гарантийной поддержки и микрофинансирования малого и среднего предпринимательства в Российской Федерации в 2015-2018 годах и первом полугодии 2019 года» показала неэффективность использования вложенных 80-ти миллиардов рублей. Как отметил пред- 


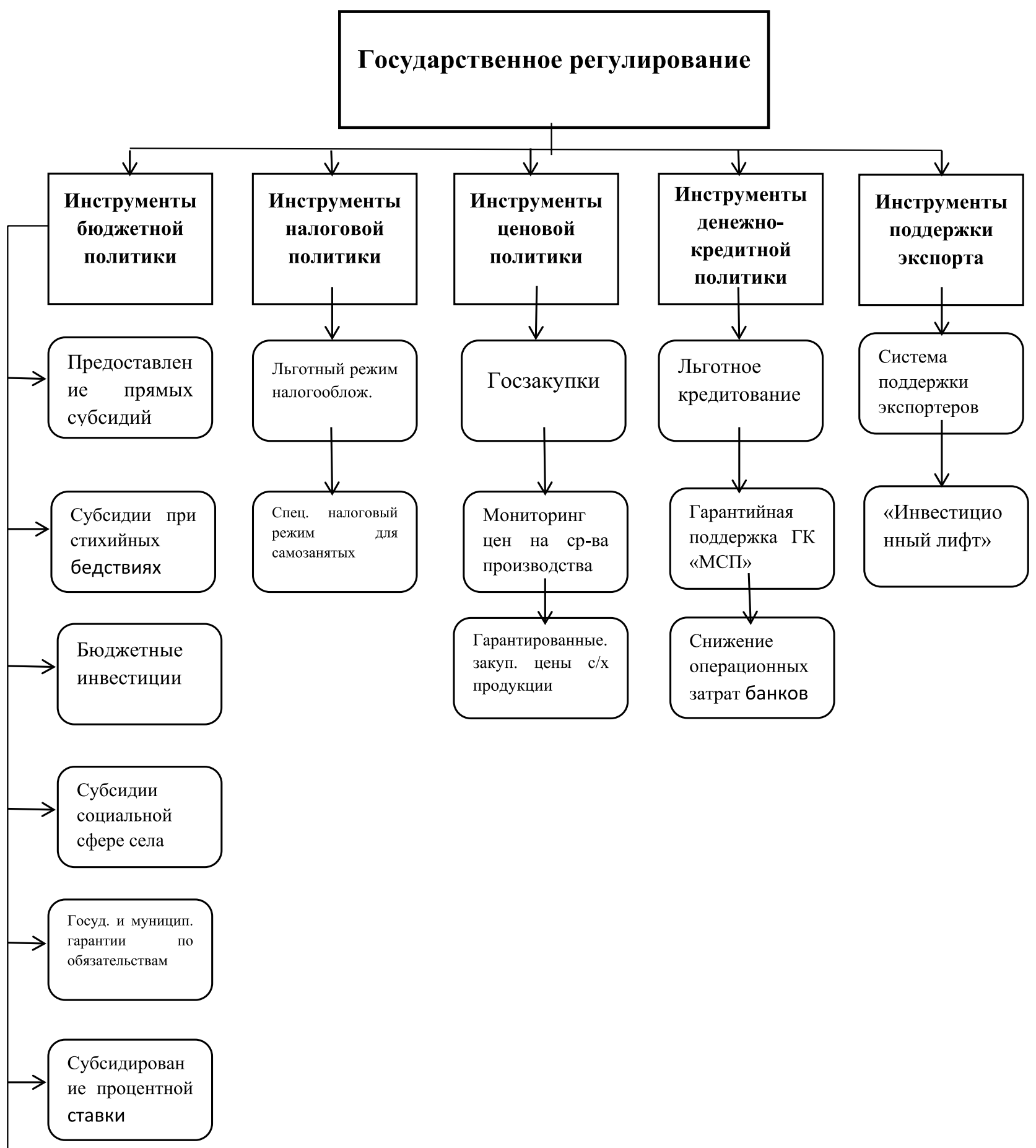

Рис. 1. Государственное регулирование и формы поддержки в секторе малого и среднего предпринимательства

седатель палаты А. Кудрин, «эта тема покрывает целый национальный проект и нас всех интересует, произойдет когда-то в этой сфере перелом или нет. Пока не происходит и даже не просматривается ...» [4].

За 9 месяцев 2019 г. в стране открылось 216459 коммерческих организаций, а прекратило свою деятельность - почти 500 000. В Краснодарском крае, например, имело место почти троекратное превышение закрывшихся бизнесов, в Ростовской области - двукратное. В Волгоградской области закрылось 10 тысяч предприятий. Причинами стали снижение покупательской активности, отсутствие местных магазинов для сбыта своей продукции, ужесточение давления контрольно-надзорных органов. 
Аналитиками отмечается, что у предпринимателей превалируют пессимистические настроения, которые наслаиваются на низкую склонность начинать свой бизнес в условиях экономической неопределенности. Согласно исследования ВЦИОМ, хотели бы иметь свое дело $76 \%$ россиян, но они боятся бедности, налогов и новых законов. Большинство (71\%) предпринимателей не считают условия для бизнеса в России благоприятными. Только $15 \%$ россиян верят, что государство принимает активные меры для развития бизнеса [5].

Наряду с особенностями менталитета населения, а также в совокупности с его невысокой финансовой грамотностью, есть и другие негативные факторы, сдерживающие деловую активность в секторе малого и среднего предпринимательства. Среди них - повышение НДС, сокращение льгот и преференций, инфляционные ожидания, регресс в увеличении реальных доходов населения, усиление позиций госкорпораций в экономике и некоторые другие.

\section{$\triangle$ Оступность креАитов}

Очевидно, что условия развития бизнеса наряду с благоприятной макроэкономической и правовой средой, наличием действенных механизмов защиты прав собственности во многом определяются доступностью источников финансирования и, в частности, заемных средств. К числу сохраняющихся проблем в этой области относятся:

- высокая стоимость кредита для субъектов МСП по сравнению с другими сегментами бизнеса;

- низкая скорость принятия решений, высокие операционные издержки при кредитовании субъектов МСП;

- недостаточное использование инструмента лизинга предприятиями МСП;

- низкая доступность заемных средств для начинающих предпринимателей;

- низкая вовлеченность субъектов МСП в закупки у крупнейших заказчиков; недостаточное использование биржевых инструментов для финансирования;

- высокий процент отказов банков ввиду недостаточности сведений о субъектах МСП.

\section{Государственное

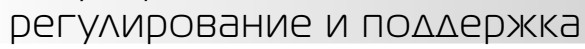

В этих условиях весьма своевременным является принятие и осуществление, начиная с 2018 г. и на период до 2024 г., ряда федеральных проектов и программ (помимо уже упомянутых выше): «Улучшение условий ведения предпринимательской деятельности», «Расширение доступа субъектов МСП к финансовым ресурсам, в том числе к льготному финансированию», «Акселерация субъектов малого и среднего предпринимательства», «Создание системы поддержки фермеров и развитие сельской кооперации» и «Популяризация предпринимательства». Принятие этих документов направлено на урегулирование взаимоотношений между бизнесом, государством, различными фондами и корпорациями в целях их упорядочения и совершенствования регулирования поддержки малого предпринимательства.

Формы государственной поддержки являются частью более широкой системы государственного регулирования и касаются применения экономических методов и инструментов с целью развития кредитования в секторе малого и среднего предпринимательства. Они включают льготы по налогообложению, субсидии, гарантийную поддержку, государственные закупки и цены, льготы по кредитованию, бюджетные инвестиции и др. (см. рис. 1).

Как отмечалось выше, в стране реализуется национальный проект «Малое и среднее предпринимательство и поддержка индивидуальной предпринимательской инициативы», который сопровождает ряд федеральных и региональных программ поддержки. Конкретными формами поддержки субъектов МСП в рамках реализуемых Программ являются:

ф финансовая поддержка,

- имущественная поддержка,

- информационно-консультационная поддержка.

\section{Программа субси ирования} $\triangle \mathrm{OXO} \triangle \mathrm{OB}$ баНКОВ

Принимая во внимание, что уровень процентной ставки по кредитам предприятиям МСП в рамках реализуемой программы ниже среднерыночных условий, в рамках национального проекта предусмотрена программа субсидирования недополученных доходов уполномоченными банками.

Ключевыми условиями субсидирования для уполномоченных банков являются:

1. субсидии предоставляются на возмещение недополученных доходов по кредитам, выданным в 2018 году субъектам МСП на реализацию проектов (на инвестиционные цели или на пополнение оборотных средств) в приоритетных отраслях по льготной ставке - не более $6,5 \%$ годовых;

2. в рамках программы субсидирования уполномоченный банк предоставляет заемщику:

1. инвестиционный кредит на реализацию проекта в приоритетных отраслях в размере от 3 млн. рублей до 1 млрд. руб. на срок до 10 лет; 
Таблица 1. Диапазон дифференцирования субсидий уровня процентной ставки для уполномоченных банков

\begin{tabular}{|l|l|}
$\begin{array}{l}\text { Диапазон объемов кредитования в рамках } \\
\text { Программы льготного кредитования } \\
\text { субъектов МСП }\end{array}$ & $\begin{array}{l}\text { Ставка субсидирования для } \\
\text { данного объема,\% }\end{array}$ \\
\begin{tabular}{|l|l|} 
В пределах 8\% от объема кредитования \\
субъектов МСП за прошлый год \\
(по данным Банка России)
\end{tabular} & 2,5 \\
\hline $\begin{array}{l}\text { Для объема свыше 8\% и до достижения 16\% от объема кредитования субъектов МСП за прошлый } \\
\text { год (по данным Банка России) }\end{array}$ & 3,0 \\
\hline $\begin{array}{l}\text { Для объема свыше 16\% и до достижения 25\% } \\
\text { от объема кредитования субъектов МСП } \\
\text { за прошлый год (по данным Банка России) }\end{array}$ & 3,5 \\
\hline
\end{tabular}

2. кредит на пополнение оборотных средств на реализацию проекта в приоритетных отраслях в размере от 3 млн. руб. до 100 млн. руб. на срок до 3 лет.

Размер субсидии уровня процентной ставки для уполномоченных банков колеблется в диапазоне от 2,5 до 3,5\%. Дифференциация ставки субсидирования поставлена в зависимость от объема кредитования, как это показано в табл. 1.

По оценкам Банка России, программа субсидирования процентных ставок пока не заработала в полную силу. При этом на то есть, по крайней мере, две причины:

- региональным банкам с базовой лицензией пока сложно выстроить устойчивые модели кредитования малого бизнеса;

- сектор МСП пока еще недостаточно развивается в силу ряда сдерживающих факторов.

\section{Корректируюшие меры}

На наш взгляд, для активизации деловой активности со стороны уполномоченных банков и заемщиков необходимо рассмотреть возможность принятия ряда корректирующих мер. В частности, целесообразно следующее:

- расширить перечень отраслей и, соответственно круг предприятий, работающих в этих сферах, с целью их вовлечения в реализацию программы льготного кредитования.

В частности, в сферу потенциальных новых субъектов участников программы включить микропредприятия, такие, например, как маленькие магазины, рестораны и гостиничный, арендный бизнес, что позволило бы тысячам новых компаний МСП получить доступ к кредитным ресурсам банков, включая онлайн-кредиты;

- в случае признания предоставленного заемщику кредита проблемным рассмотреть вопрос о раз- решительной практике проведения банком рефинансирования таких кредитов, поскольку наличие признаков проблемности ссуды еще не является признаком того, что заемщик не вернет ее;

- исключить при отборе банков в группу уполномоченных такие дополнительные критерии, как прирост кредитного портфеля малого и среднего бизнеса и объем этого портфеля в совокупном корпоративном портфеле. Наличие этих критериев не является гарантией отражения положительного опыта работы банка с данной категорией заемщиков, свидетельствуя лишь о взаимодействии, а не результате взаимодействия;

- более активно вовлекать в реализацию Программы банки с базовой лицензией, которые, как правило, работают с данной категорией заемщиков. Снятие дополнительных критериев для отбора банков позволит расширить круг участников Программы и доступность предприятий МСП к финансовым услугам;

- повысить уровень норматива Н6 для банков с базовой лицензией до $25 \%$, то есть на 5 процентных пунктов, что позволит расширить объем кредитной поддержки субъектов МСП на региональном уровне;

- подкрепить процесс предоставления субсидий по процентной ставке уполномоченным банкам более гибкой шкалой оценки кредитных рисков субъектов МСП, уровень которых в данном сегменте кредитования остается высоким, что ограничивает поддержку малого и среднего предпринимательства;

- рассмотреть вопрос об освобождении от налогообложения предоставленных субсидий и льгот.

\section{Совершенствование механизма креАитования МСП}

Не менее важным фактором облегчения доступности кредитных ресурсов для предприятий МСП является 
Таблица 1. Диапазон дифференцирования субсидий уровня процентной ставки для уполномоченных банков

\begin{tabular}{l|l|}
\hline $\begin{array}{l}\text { Диапазон объемов кредитования в рамках } \\
\text { Программы льготного кредитования } \\
\text { субъектов МСП }\end{array}$ & $\begin{array}{l}\text { Ставка субсидирования для } \\
\text { данного объема,\% }\end{array}$ \\
\begin{tabular}{|l|l|} 
В пределах 8\% от объема кредитования \\
субъектов МСП за прошлый год \\
(по данным Банка России)
\end{tabular} & 2,5 \\
\hline $\begin{array}{l}\text { Для объема свыше 8\% и до достижения 16\% от объема кредитования субъектов МСП за прошлый } \\
\text { год (по данным Банка России) }\end{array}$ & 3,0 \\
\hline $\begin{array}{l}\text { Для объема свыше 16\% и до достижения 25\% } \\
\text { от объема кредитования субъектов МСП } \\
\text { за прошлый год (по данным Банка России) }\end{array}$ & 3,5 \\
\hline
\end{tabular}

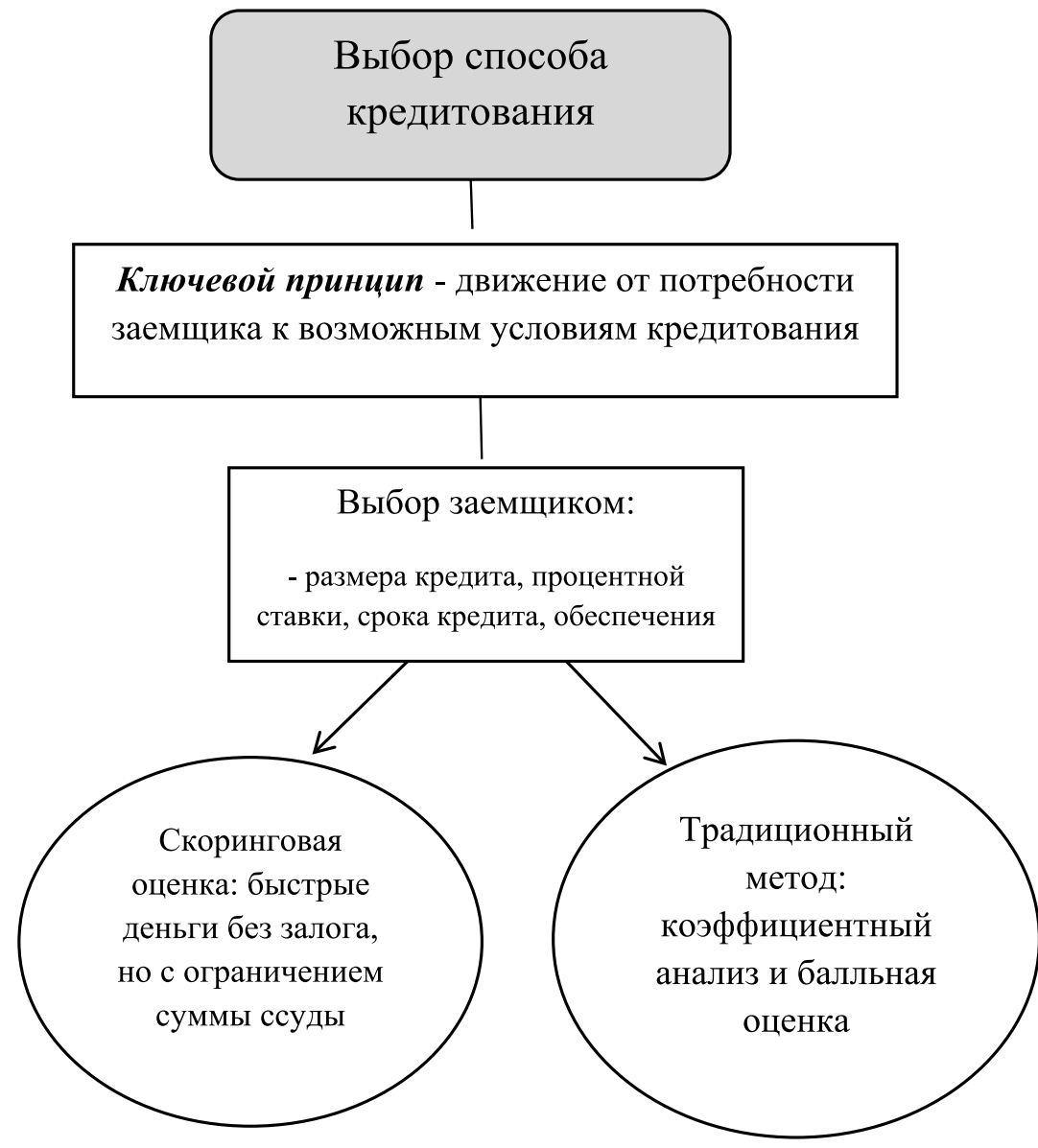

Рис. 2. Выбор метода кредитования предприятия МСП

дальнейшее совершенствование механизма их кредитования.

Известно, что в ряду проблем банковского кредитования МСП одними из основных являются следующие:

Для банка:

- неудовлетворительное финансовое состояние фирмы;
- непрозрачность ведения бизнеса этого сегмента предпринимательской деятельности;

- проблемы с экономической и юридической грамотностью многих руководителей малых предприятий;

- отсутствие ликвидных залогов и др.

Для заемщика:

- высокие процентные ставки; 
- жесткие условия и недостаток информации при получении кредита;

- требование ликвидного залога;

- длительные сроки рассмотрения заявки и др.

В целях совершенствования механизма кредитования предприятий МСП, обеспечения его соответствия потребностям заемщиков и расширению их доступа к кредитным ресурсам, повышения качества и доходности кредитного портфеля считаем целесообразным, во-первых, внести изменения в алгоритм механизма кредитования предприятий МСП, во-вторых, шире использовать маркетинговые исследования в целях установления персонифицированных процентных ставок, а также снижения риска невозвратов по кредитам.

Предлагаемый алгоритм учитывает опыт банков в области кредитования малого и среднего бизнеса в России и за рубежом. Центральный принцип алгоритма - движение от потребностей заемщика к возможным условиям кредитования (см. рис. 2). В результате создается конкурентоспособный и качественный кредитный продукт, предназначенный для малых и средних предприятий.

Возможны два варианта получения кредита. Чтобы определить подходящий вариант, предприниматель самостоятельно определяет для себя размер кредита, процентную ставку, по которой он готов кредитоваться, срок кредита и обеспечение. В зависимости от этих критериев определяется один из двух вариантов.

І вариант. Проведение скоринговой оценки предприятия МСП с возможностью получить быстрые деньги без залога, но размер кредита будет ограничен. Целесообразно установить порог суммы кредита, ниже которой будет применяться данная методика при обращении заемщика в банк. При этом лояльный подход банка будет компенсирован увеличением процентной ставки.

Программа скоринга разрабатывается совместно специалистами аналитического отдела, отдела привлечения и кредитного отдела банка и учитывает массив факторов, основные из которых - размер кредита, отрасль, в которой работает предприятие, срок его работы, финансовые показатели, кредитная история и объем действующих кредитов при их наличии. Учитывается история и продолжительность сотрудничества предприятия с банком, включая как кредитование, так и обороты по счетам.

II вариант. Традиционный метод, при котором собирается полный пакет документов с последующим анализом специалиста аналитического/кредитного подразде- ления банка и принятием решения о выдаче кредита или об отказе в кредитовании.

Маркетинговые исследования в целях установления персонифицированных процентных ставок могут охватывать 3 этапа.

I этап. Проведение клиентскими менеджерами банка маркетингового исследования в целях сегментации МСП соответствующей территории по видам деятельности для определения перспектив развития предприятий. При этом меняющиеся рыночные условия подлежат постоянному мониторингу. В случае если выбранное направление бизнеса развивается достаточно динамично, то кредитные ресурсы следует направлять в данную часть рынка.

II этап. Установление дифференцированной процентной ставки по сегментам рынка, выявленным на предыдущем этапе. В нынешней практике, к сожалению, происходит усреднение рисков невозврата ссуды для всех заемщиков, что неверно. В менее рентабельном бизнесе необходимы более тщательная финансовая оценка заемщиков и увеличение процентных ставок в целях покрытия возможных потерь.

III этап. Привлечение к кредитованию предприятий СМП, функционирующих в выявленных секторах рынка. В интересах привлечения большего количества заемщиков необходимо обеспечить:

- размещение рекламы в специализированных для данного бизнеса изданиях;

- разработку схем кредитования, которые бы учитывали специфику конкретного бизнеса;

- снижение процентных ставок для рентабельных бизнесов.

\section{Выво $\triangle$}

Государственное регулирование и формы поддержки в сегменте российского малого и среднего предпринимательства, а также дальнейшее совершенствование механизма кредитования предприятий МСП - ключевые факторы преодоления признаков стагнации сектора. В части развития нормативной базы решению данной проблемы могла бы способствовать реализация комплекса мер, в частности, включение микропредприятий в сферу потенциальных участников программы льготного финансирования. В рамках модернизации механизма кредитования заемщиков сегмента МСП целесообразно центральным принципом его алгоритма считать движение от потребностей заемщика к возможным условиям кредитования, а также шире использовать маркетинговые исследования для оптимизации каналов вложения ресурсов и установления персонифицированных процентных ставок по кредиту. 


\section{ЛИТЕРАТУРА}

1. А. Фейнберг. Росстат впервые раскрыл долю малого и среднего бизнеса в экономике / А. Фейнберг. - Текст: электронный // Информационное агентство «РБК»: [сайт].—URL: https://www.rbc.ru/economics/05/02/2019/5c5948c59a794758389cfdf7 (дата публикации: 05.02.2019).

2. С. Р. Борисов. Почему малый бизнес еще не стал драйвером экономического роста в России? // Бизнес. Общество. Власть. 2018. № 2 (28). С. 49-52.

3. Сайт «rbc.ru». [Электронный ресурc]. URL: https://www.rbc.ru/economics/07/11/2019/5dc191aa9a7947de940ab259/

4. Сайт «tass.ru». [Электронный ресурc]. URL: https://tass.ru/msp/7419145.

5. А. Хандриков. Итоги 2019 года для малого и среднего предпринимательства / А. Хандриков. — Текст электронный // Яндекс Дзен: [сайт]. — URL: https:// zen.yandex.ru/media/id/5bd7430532са1533928ee0ad/itogi-2019-goda-dlia-malogo-i-srednego-predprinimatelstva-5e142d600be00a00afbcb332 (дата публикации: 07.01.2020).
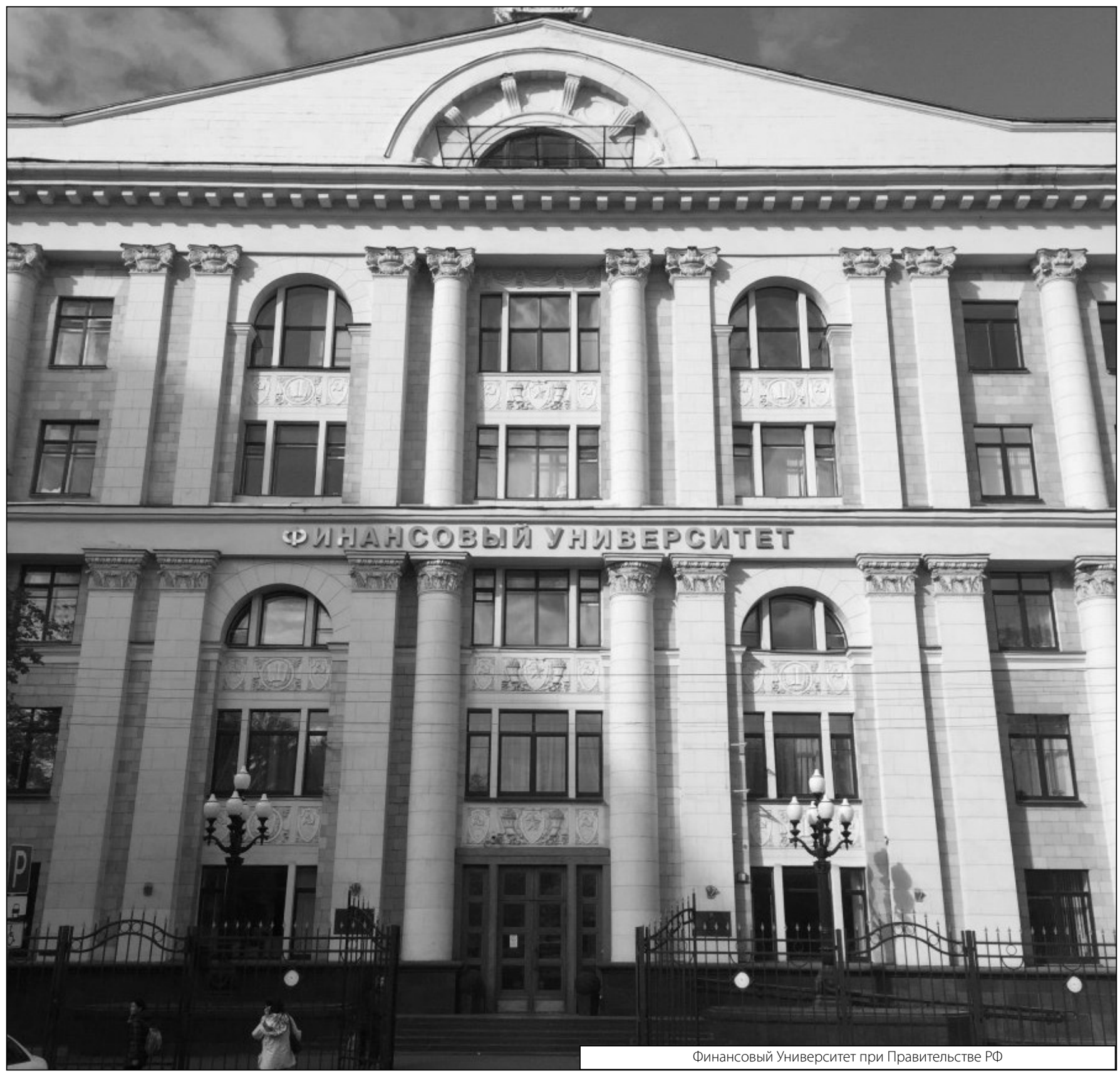